УДК 621.315 .592

\title{
Остаточные напряжения в кремнии и их эволюция при температурной обработке и облучении
}

\author{
(C) И.Е. Матяш ${ }^{1}$, И.А. Минайлова ${ }^{1, \llbracket, ~ Б . К . ~ С е р д е г а ~}{ }^{1}$, Л.И. Хируненко \\ ${ }^{1}$ Институт фризики полупроводников им. В.Е. Лашкарева Национальной академии наук Украины, \\ 03028 Киев, Украина \\ ${ }^{2}$ Институт фризики Национальной академии наук Украины, \\ 03028 Киев, Украина \\ I E-mail: irinaminailiva125@gmail.com
}

(Получена 20 января 2017 г. Принята к печати 6 февраля 2017 г.)

\begin{abstract}
Исследованы распределения внутренних механических напряжений нелегированного и легированного оловом кремния и влияния на них облучения электронами с энергией $5 \mathrm{MэB} \mathrm{и} \mathrm{температурной} \mathrm{обработки}$ при $450^{\circ}$ С. Измерения напряжений проводились методом, основанным на регистрации двулучепреломления с помощью модуляционной поляриметрии. Показано, что легированный оловом кремний имеет полосы точечных дефектов с неоднородным распределением остаточных напряжений до 20 кг/см². Температурная обработка при $450^{\circ} \mathrm{C}$ приводит к повышению остаточных напряжений в образце до 50 кг $/ \mathrm{cm}^{2}$. Выявлено, что радиационные дефекты, которые образовались при облучении кремния, легированного оловом, приводят к уменьшению остаточных напряжений до $2-3$ кг/ $\mathrm{cm}^{2}$.
\end{abstract}

DOI: 10.21883/FTP.2017.09.44876.8527

\section{1. Введение}

Кремний $(\mathrm{Si})$, благодаря своим электрофизическим и механическим свойствам, будет оставаться основным материалом для производства элементной базы современной электроники еще долгое время [1-3]. Свойства полупроводника, как известно, определяются его дефектно-примесным составом. Одним из перспективных методов управления параметрами кремния является легирование изовалентными примесями $[4,5]$. Изовалентные примеси являются электрически нейтральными и практически не влияют на выходные электрические параметры материала. Влияние изовалентных примесей на свойства $\mathrm{Si}$ определяется в основном внутренними упругими деформациями решетки, которые возникают вследствие разницы ковалентных радиусов атомов матрицы и примеси. Присутствие внутренних локальных деформаций, как известно, может существенно влиять на процессы дефектно-примесного взаимодействия как при выращивании кристалла, так и при различных внешних воздействиях. В поле упругих деформаций может происходить изменение процессов диффузии примесей и дефектов, кроме того, атомы изовалентных примесей могут непосредственно взаимодействовать с собственными вакансиями и междоузлиями $\mathrm{Si}$, тем самым меняя дефектно-примесное взаимодействие. Поэтому даже небольшие изменения механических напряжений, возникающие в кремнии под действием различных внешних факторов (облучение, термообработка), могут приводить к изменению электрофизических свойств материала. Следует отметить, что аналогичные изменения могут возникать как в монокристаллическом кремнии, так и в нанослоях. Поэтому изучение и анализ остаточных механических напряжений как в выращенных материалах, так и в подвергнутых таким внешним воздействиям, как облучение и термообработка, является актуальным. Такие исследования представляют интерес как для теоретиков, так и для технологов-разработчиков приборов твердотельной электроники. Механические и прочностные характеристики полупроводниковых материалов (микротвердость, трещиностойкость и микрохрупкость) в процессе облучения изучены достаточно хорошо [6]. В то время как сведений о диагностике остаточных механических напряжений в $\mathrm{Si}$ до и после облучения и влиянии на них легирования изовалентными примесями имеется недостаточно.

\section{2. Методика эксперимента}

В работе исследовались образцы кремния $(\mathrm{Si})$ и кремния, легированного оловом $\mathrm{Si}(\mathrm{Sn})$, вырезанные из слитков кремния $n$-типа проводимости (легированных фосфором), выращенных методом Чохральского. Концентрация олова в образцах составляла $(1-2) \cdot 10^{19} \mathrm{~cm}^{-3}$. Содержание кислорода $N_{\mathrm{O}}$ в образцах определялось по интенсивности полосы $\sim 1107 \mathrm{~cm}^{-1}$ при комнатной температуре и составляло $(5-6) \cdot 10^{17} \mathrm{~cm}^{-3}$. Концентрация углерода, оцененная по полосе поглощения $607 \mathrm{~cm}^{-1}$, изменялась в пределах $(1-1.8) \cdot 10^{17} \mathrm{~cm}^{-3}$. Линейные размеры всех образцов были $\sim X \times Y \times Z=$ $=1 \times 0.6 \times 0.25 \mathrm{~cm}$. При этом наибольший размер был перпендикулярен направлению роста слитков [111].

Образцы $\mathrm{Si}(\mathrm{Sn})$ подвергались либо термообработке при $T=450^{\circ} \mathrm{C}$ в течение 40 ч $-\mathrm{Si}(\mathrm{Sn})_{t}$, либо облучению на линейном ускорителе электронов с энергией 5 МэВ при комнатной температуре. Доза облучения была $5 \cdot 10^{17} \mathrm{~cm}^{-2}-\mathrm{Si}(\mathrm{Sn})_{i r}$. 


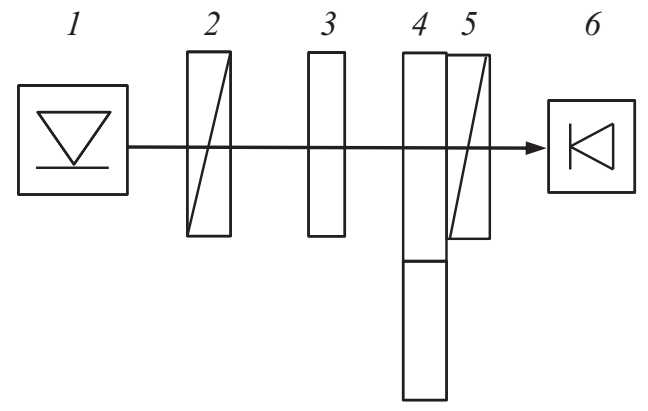

Рис. 1. Оптическая схема для измерения остаточных напряжений: 1 - лазер, 2 - поляризатор, 3 - образец, 4 - модулятор поляризации, 5 - поляризатор, $6-$ фотодиод.

Для диагностики остаточных напряжений использовалась регистрация двулучепреломления методом модуляционной поляриметрии (МП) [7]. Оптическая схема установки показана на рис. 1. Излучение лазера 1 $(\lambda=1.15$ мкм) проходит через линейный поляризатор 2 , азимут которого выставляется таким образом, чтобы плоскость поляризации линейно поляризованного излучения, направляемого на образец 3 , находилась под углом $45^{\circ}$ к его осям $X$ и $Y$. В образце за счет линейного двулучепреломления излучение преобразуется в эллиптически поляризованное и через модулятор поляризации 4 и линейный поляризатор 5 направлено на фотодиод 6. Модулятор поляризации 4 совместно с поляризатором 5, оси которого находятся под углом $45^{\circ}$ относительно осей модулятора, являются динамическим анализатором состояния поляризации света. Он позволяет определить интенсивность циркулярной и линейной компоненты эллиптически поляризованного света отдельно, поскольку сигналы пропорциональные указанным интенсивностям регистрируются на разных частотах [8]. Мерой анизотропии является интенсивность циркулярной компоненты излучения, которая измерялась на частоте модуляции:

$$
I_{V}=E_{X} \cdot E_{Y} \cdot \sin \Delta \varphi .
$$

Разность фаз $\Delta \varphi$ между линейно поляризованными волнами $E_{X}$ и $E_{Y}$ на выходе из образца равна:

$$
\Delta \varphi=(2 \pi / \lambda) \Delta n d,
$$

где $\lambda-$ длина волны сканирующего излучения и $d-$ толщина образца. Анизотропию показателя преломления $\Delta n$ можно представить как

$$
\Delta n=n_{X}-n_{Y}=C\left(\sigma_{X}-\sigma_{Y}\right),
$$

где $n_{X}, n_{Y}$ и $\sigma_{X}, \sigma_{Y}-$ показатели преломления и напряжения вдоль соответствующих направлений, а $C-$ константа Брюстера.

Таким образом, измеренная интенсивность циркулярной составляющей излучения пропорциональна величине внутренних напряжений $\left(\sigma_{X}-\sigma_{Y}\right)$, и путем сканирования $X-Y$ поверхности образцов в работе были зарегистрированы координатные распределения величины напряжений. Установка позволяет регистрировать сигнал, пропорциональный значению $\left(\sigma_{X}-\sigma_{Y}\right)$ в относительных единицах. Тем не менее распределение напряжений представлено в абсолютных единицах, так как установка была откалибрована внешним мерным усилием, а ее разрешающая способность была определена на уровне $10 \Gamma / \mathrm{cm}^{2}$.

\section{3. Результаты и обсуждение}

Распределение измеренных остаточных механических напряжений в образце исходного кремния $-\mathrm{Si}_{\text {niti }}$, пропускание которого составляло $T=48 \%$, показано на рис. 2. На рисунке положительный знак соответствует сжатию вдоль $O Y$, а отрицательный - растяжению вдоль этой оси. Основными источниками остаточных напряжений в кремнии являются дефекты структуры, примеси и ростовые дефекты. Вклад в напряжения, как известно, может также вносить конденсация вакансий, концентрация которых на границе раздела расплав-кристалл достаточно высокая [9]. Полосы точечных дефектов могут возникать в кристалле вследствие наличия переменных градиентов температуры, существующих в кристалле во время роста [10]. Любое отклонение от постоянного градиента температуры на фронте кристаллизации вызывает неоднородное термическое расширение, приводящее к образованию внутренних механических напряжений в затвердевающем кристалле [11]. Из рисунка видно, что приповерхностные напряжения значительно отличаются от остаточных напряжений в средней части пластины кремния. Следовательно, можно сделать вывод о равномерном распределении неоднородностей в слитке и о том, что основным механизмом возникновения напряжений в образце $\mathrm{Si}_{\text {init }}$ является неоднородное распределение дефектов, возникших при обработке его поверхностей.

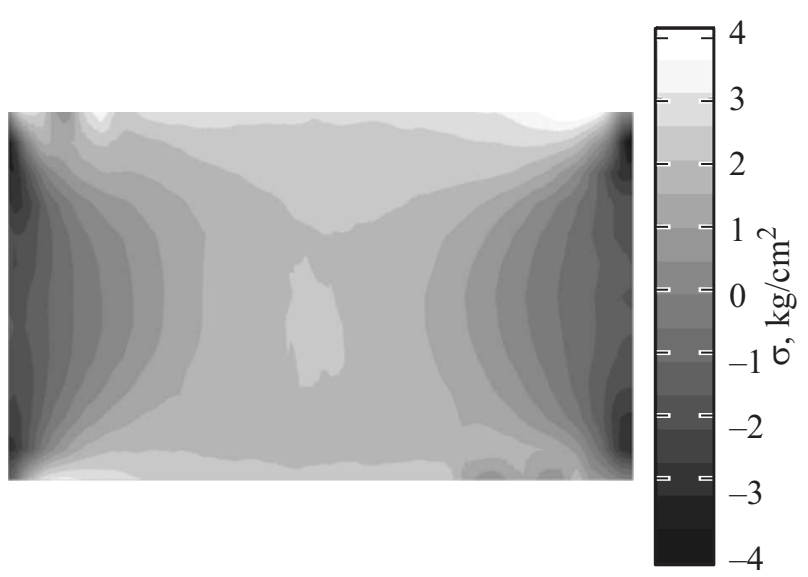

Рис. 2. Распределение остаточных напряжений в образце исходного кремния $n$-типа. 
При измерении механических напряжений в образце кремния, легированного оловом - $\mathrm{Si}(\mathrm{Sn})$, было обнаружено (рис. 3), что пластина имеет характерные полосы роста или полосы точечных дефектов. Может быть несколько причин возникновения полосатости распределения дефектов. Большинство исследователей, как известно, основную роль в образовании полосатости распределения дефектов отводят атомам кислорода [12]. Вероятной причиной наблюдаемого распределения дефектов может быть установленное ранее коррелированное распределение атомов олова и кислорода в решетке $\mathrm{Si}$ [13]. Атомы олова имеют тенденцию при выращивании располагаться в ближайших координационных сферах относительно кислорода, что может приводить к наблюдаемому эффекту. Одной из основных причин возникновения полосатости в исследуемых образцах может быть также негомогенность распределения олова по образцу. Возникновение полос точечных дефектов и неоднородного распределения примеси сопровождается уменьшением пропускания образца $\mathrm{Si}(\mathrm{Sn})$ до $T=39 \%$ по сравнению с нелегированным оловом кремнием.

Любое внешнее воздействие, приводящее к изменению распределения дефектов в кристалле, будет приводить к изменению величины и координатной зависимости механических напряжений. При высокотемпературных обработках кремния основным механизмом изменения механических напряжений является образование термодоноров. При использованной в работе обработке при $450^{\circ} \mathrm{C}$ процессы массопереноса и преципитации кислорода лимитируются обычной (нормальной) диффузией межузельных атомов $\mathrm{O}_{i}$ [14]. Происходит распад пересыщенного раствора кислорода с образованием и ростом зародышей новой объемной фазы, так называемых кислородных преципитатов, или термодоноров, представляющих собой частицы окисного соединения вида $\mathrm{SiO}_{x}[15-17]$. Образование термодонорных центров коррелирует с уменьшением концентрации межузельно-

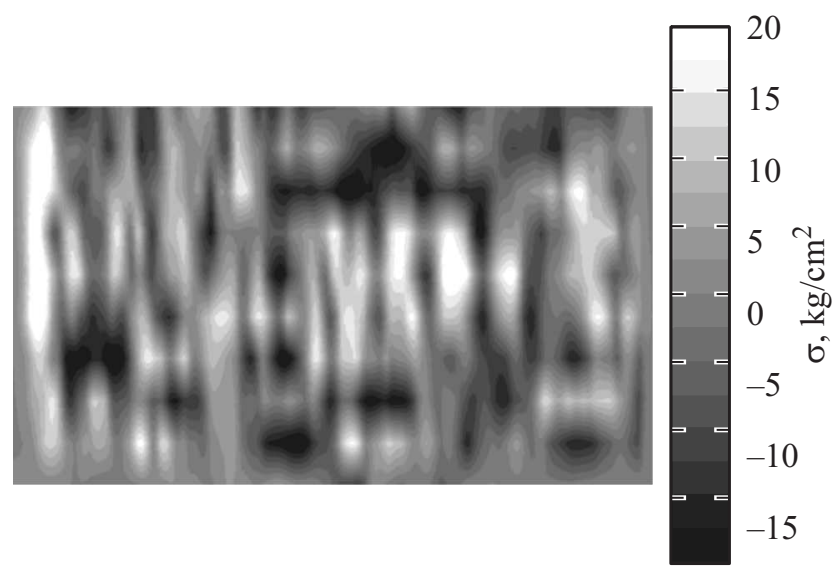

$-20$

Рис. 3. Распределение остаточных напряжений в образце кремния, легированного оловом. Концентрация олова $(1.9) \cdot 10^{19} \mathrm{~cm}^{-3}$.

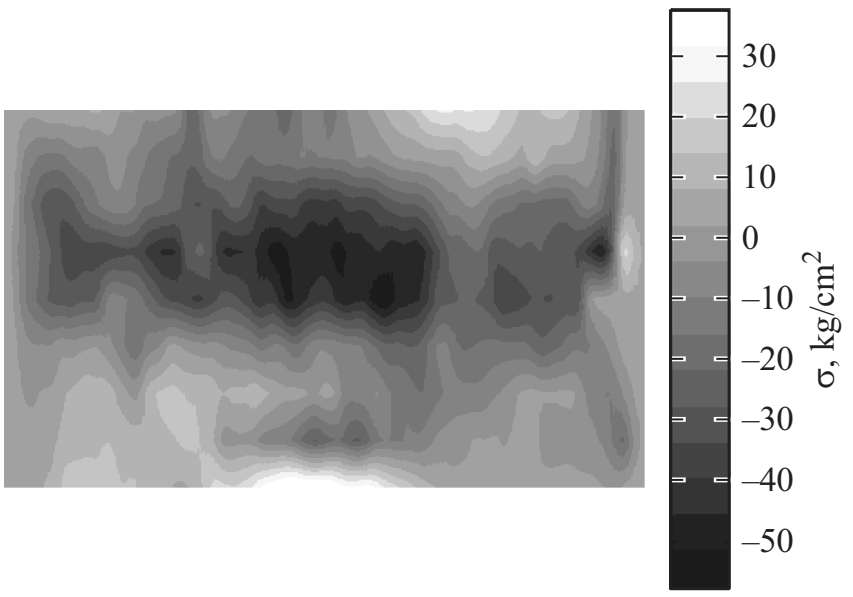

Рис. 4. Распределение остаточных напряжений в образце кремния, легированного оловом, после температурной обработки при $450^{\circ} \mathrm{C}$ в течение 40 ч.

го кислорода $\mathrm{O}_{i}$. На рис. 4 показано распределение остаточных напряжений в образце кремния, легированного оловом, после температурной обработки $\mathrm{Si}(\mathrm{Sn})_{t}$. Термообработка производилась при $450^{\circ} \mathrm{C}$ в течение 40 ч. Перед термообработкой образцы подвергались отжигу при $900^{\circ} \mathrm{C}$ в течение 20 мин для более равномерного распределения кислорода по образцу. Из рисунка видно, что остаточные напряжения в образце кремния после обработки $450^{\circ} \mathrm{C}$ увеличились в $\sim 2.5$ раза (до 50 кг $/ \mathrm{cm}^{2}$ ) при уменьшении пропускания до $T=34 \%$. Это объясняется тем, что формирование кислородсодержащих термодоноров происходит путем последовательного присоединения определенных структурных единиц к центрам зарождения термодоноров. В результате введения таких преципитатов в кристаллах увеличиваются поля упругих напряжений. Кроме того, во время термообработки диффундирующий кислород может локализоваться вблизи атомов олова, концентрация которого в исследуемом образце превышает содержание кислорода, что также приведет к увеличению напряжений в решетке. Также можно утверждать, что распределение дефектов в образце при обработке становится более равномерным, так как не наблюдаются напряжения, вызванные полосами точечных дефектов. Наблюдаемая в его центре область растяжения, по всей вероятности, является областью сосредоточения дефектов-преципитатов $\mathrm{SiO}_{x}$, которые имеют элементарный объем ячейки больше, чем атом кремния $\mathrm{Si}$.

В работе также были исследованы остаточные напряжения в образце кремния, легированного оловом после облучения его электронами с энергией $5 \mathrm{MэB}$, облученного при комнатной температуре и отожженного до $300^{\circ} \mathrm{C} \mathrm{Si}(\mathrm{Sn})_{i r}$ (рис. 5). Из рисунка видно, что остаточные напряжения распределены практически без полосчатой картины по образцу и уменьшились примерно в 10 раз по абсолютному значению по сравнению с напряжениями в необлученном $\mathrm{Si}(\mathrm{Sn})$. При этом 


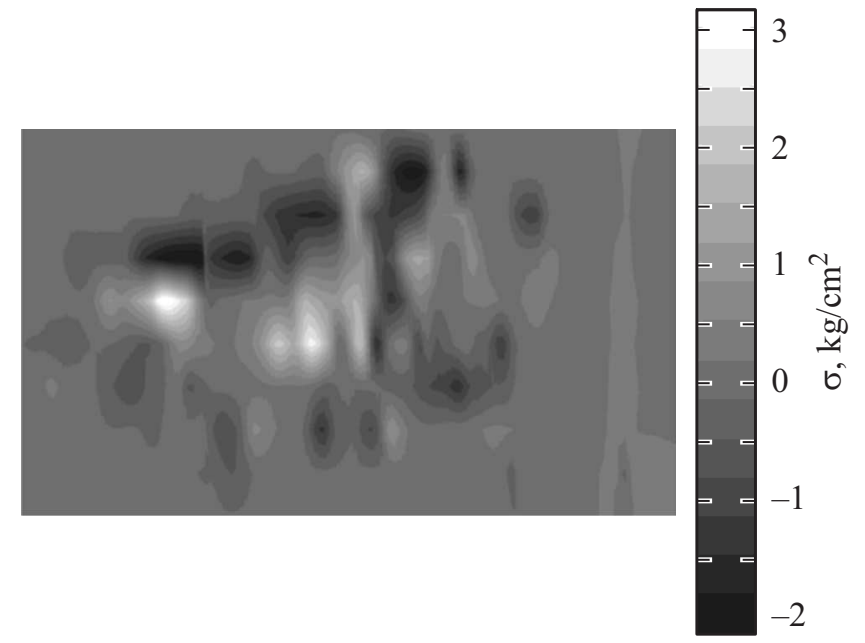

Рис. 5. Распределение остаточных напряжений в образце кремния, легированного оловом, после облучения электронами с энергией 5 МэВ. Доза облучения $5 \cdot 10^{17} \mathrm{~cm}^{-2}$.

пропускание образца составляет $T=20 \%$. Наблюдаемый эффект, по-видимому, можно объяснить следующим образом. Ранее методом ИК фурье-спектроскопии были исследованы спектры поглощения радиационных дефектов образцов $\mathrm{Si}(\mathrm{Sn})[18,19]$. Согласно этим исследованиям, в $\mathrm{Si}(\mathrm{Sn})$, подвергнутых облучению и последующей термообработке при $300^{\circ} \mathrm{C}$, наблюдаются следующие радиационные дефекты: вакансия + кислород (VO), VO, локализованные на атоме олова $(\mathrm{SnVO})$, комплексы $\mathrm{Sn}_{2} \mathrm{~V}_{2}$ и центры межузельный углерод-межузельный кислород $\left(\mathrm{C}_{i} \mathrm{O}_{i}\right)$. По нашему мнению, именно этот образовавшийся при облучении и последующем отжиге ряд радиационных дефектов межузельного и вакансионного типа приводит к небольшим остаточным напряжениям. Таким образом, локализация диффундирующих при облучении или последующем отжиге собственных вакансий и межузельного углерода способствует уменьшению остаточных напряжений в решетке. Как видно из рис. 5, они имеют абсолютные значения в пределах

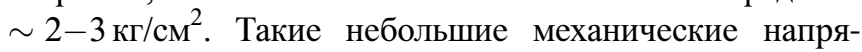
жения и низкое пропускание свидетельствуют о том, что концентрация дефектов, обладающих напряжениями сжатия, сравнима с концентрацией дефектов, создающих в решетке напряжения растяжения, что и приводит к исчезновению полосчатости.

\section{4. Заключение}

Таким образом, в работе методом модуляционной поляриметрии было экспериментально проанализировано распределение дефектов в образцах нелегированного и легированного оловом Чохральского кремния $n$-типа проводимости. Также исследованы влияния облучения и высокотемпературной обработки на остаточные напряжения в решетке кремния. Применение метода МП позволило идентифицировать остаточные напряжения в образцах в абсолютных единицах с разрешающей способностью 10 г/ $\mathrm{cm}^{2}$.

Было показано, что увеличение концентрации дефектов в образце может привести как к увеличению (температурная обработка), так и к уменьшению (облучение) остаточных напряжений. Это будет зависеть от нескольких факторов: от вида дефектов, их размеров относительно элементарного объема ячейки материала и равномерности распределения дефектов по объему образца. Возникновение одного типа дефектов, а именно соединения вида $\mathrm{SiO}_{x}$, при термообработке привело к увеличению остаточных напряжений. Облучение легированного кремния, в свою очередь, привело к возникновению различных типов дефектов, которые взаимно нивелировали возникшие деформации, что привело к уменьшению абсолютных значений остаточных напряжений в образце. В любом случае однородное внешнее воздействие на образец, приводящее к массопереносу, способствовало более однородному распределению в нем внутренних механических напряжений.

Практическое применение метода заключается в прогнозировании изменения остаточных напряжений, а следовательно, и других свойств кремния и микроэлектронных приборов на его основе при эксплуатации в условиях высоких температур и/или с повышенным уровнем ионизирующего излучения.

\section{Список литературы}

[1] T. Hideki. J. App. Phys., 43, 4055 (2004).

[2] S.W. Glunz, R. Preu, D. Biro. Comprehensive Renewable Energy, 1, chap. 1.16 (2012).

[3] A. Bukowski. Acta Phys. Polon. A, 124, 235 (2013).

[4] L.I. Khirunenko, O.O. Kobzar, Yu.V. Pomozov, M.G. Sosnin, M.O. Tripachko. Phys. B, 340, 541 (2003).

[5] L.I. Khirunenko, O.O. Kobzar, Yu.V. Pomozov, M.G. Sosnin, M.O. Tripachko, N.V. Abrosimov, H. Riemann. Phys. B, 340, 546 (2003).

[6] Ю.И. Головин, А.А. Дмитриевский, Н.Ю. Сучкова. ФТТ, 50, 26 (2008).

[7] L.I. Berezhinsky, I.L. Berezhinsky, O.N. Grigorev, B.K. Serdega, V.A. Ukhimchuk. J. Eur. Ceram., 27, 2513 (2007).

[8] И.Е. Матяш, Б.К. Сердега. Оптоэлектрон. и полупроводн. техн., 40, 155 (2005).

[9] V.V. Voronkov. J. Cryst. Growth., 59, 625 (1982).

[10] T. Taishi, Y. Ohno, I. Yonenaga. Phys. B, 401, 560 (2007).

[11] T. Marek, M. Werner, G. Gerth. Cryst. Res. Technol., 35, 769 (2000).

[12] М.В. Меженный, М.Г. Мильвидский, М.П. Павлов. ФТТ, 43, 1 (2001).

[13] Ю.В. Помозов, М.Г. Соснин, Л.И. Хируненко, В.И. Яшник. ФТП, 34, 1035 (2000).

[14] F. Shimura. Semicond. Semimet., 42, 191 (1994).

[15] Proc. NATO Advanced Workshop on the Early Stages of Oxygen Precipitation in Silicon (Exeter, U.K., 1996), ed. by R. Jones. NATO ASI Ser., 3. High Technology (Kluwer, Academic Publishers, Dordrecht, 1996) v. 17.

[16] V.P. Markevich, L.F. Makarenko, L.I. Murin. Mater. Sci. Forum., 589, 38 (1989). 
[17] S.A. McQuaid, M.J. Binns, C.A. Londos, J.H. Tucker, A.R. Brown, R.C. Newman. J. Appl. Phys., 77, 1427 (1995).

[18] L.I. Khirunenko, O.A. Kobzar', Yu.V. Pomozov, M.G. Sosnin, N.A. Tripachko, N.V. Abrosimov, H. Riemann. Sol. St. Phenomena, 95-96, 393 (2004).

[19] L.I. Khirunenko, O.A. Kobzar, Yu.V. Pomozov, M.G. Sosnin, N.A. Tripachko, V.P. Markevich, L.I. Murin, A.R. Peaker. Phys. Status. Solidi C, 0, 694 (2003).

Редактор Г.А. Оганесян

\section{Residual stresses and their evolution after heat treatment and irradiation in silicon}

I.E. Matyash ${ }^{1}$, I.A. Minailova ${ }^{1}$, B.K. Serdega ${ }^{1}$,

L.I. Khirunenko ${ }^{2}$

${ }^{1}$ Lashkarev Institute of Semiconductor Physics, National Academy of Sciences of Ukraine,

03028 Kyiv, Ukraine

${ }^{2}$ Institute of Physics, National Academy

of Sciences of Ukraine,

03028 Kyiv, Ukraine

Abstract Distribution of residual stresses in $\mathrm{Si}$ and tin-doped $\mathrm{Si}$ samples, grown by Czochralski method, were measured in study. Changes of the mechanical stresses appearing owing to irradiation with $5 \mathrm{MeV}$ electrons and thermal treatment at $450^{\circ} \mathrm{C}$ of these samples were investigated. Registration of the birefringence by modulation polarimetry allowed us to estimate the absolute value of the mechanical stresses. It is shown that the tin-doped sample has strip of point defects with an inhomogeneous distribution of residual stresses to $20 \mathrm{~kg} / \mathrm{cm}^{2}$. Thermal treatment of tin-doped $\mathrm{Si}$ results in an increase of residual stresses in the sample to $50 \mathrm{~kg} / \mathrm{cm}^{2}$. Defects appearing upon irradiation lead to decrease residual stresses in the sample to $2-3 \mathrm{~kg} / \mathrm{cm}^{2}$. 